\title{
A FULLY-INTEGRATED MULTI-SITE PRESSURE SENSOR FOR WIRELESS ARTERIAL FLOW CHARACTERIZATION
}

\author{
Andrew DeHennis and Kensall D. Wise \\ NSF Engineering Research Center for Wireless Integrated MicroSystems \\ Department of Electrical Engineering and Computer Science \\ The University of Michigan, Ann Arbor, MI 48109-2122 \\ ph: (734) 615-7020; email: hde@umich.edu
}

\begin{abstract}
This paper presents a fully-integrated battery-free sensing system that uses a two-site wireless pressure measurement for the detection of arterial stenosis. The remotely-powered system uses a backscatter-modulated passive-telemetry interface and transmits sensor as well as reference information to an external system. The monolithic process used to realize the system integrates a $3 \mu \mathrm{m}$ BiCMOS circuit with silicon-on-glass absolute pressure sensors and an on-chip antenna. The wireless sensor interface consumes $340 \mu \mathrm{W}$ and uses capacitance-to-frequency conversion for readout of the vacuum-sealed pressure transducers. The integrated device has a $200 \mu \mathrm{m}$ profile and a volume of $2 \mathrm{~mm}^{3}$. The system can sense a reduction in flow of $13 \%$, which corresponds to a differential pressure of $3 \mathrm{mmHg}$.
\end{abstract}

\section{INTRODUCTION}

Present stroke-prevention procedures utilize carotid artery angioplasty followed by stenting to minimize effects from plaque build-up and increase arterial flow. However, the implantation of stents can sometimes cause restenosis of the artery during the healing process. Currently, the diagnosis of restenosis requires catheterization. To replace this procedure, technology to implement sensors along with the stent itself is currently being pursued [1]. Implementing wireless sensors that can be deployed during the stenting procedure would allow transcutaneous querying of the effects of restenosis and potentially the ability to diagnose other concerns in the cardiovascular system. Implementation of this technology requires a batteryless low-profile, transduction system that can be remotely powered to take queried measurements. An illustration of such a system is shown in Figure 1.

Cardiologists primarily rely on the measurement of arterial

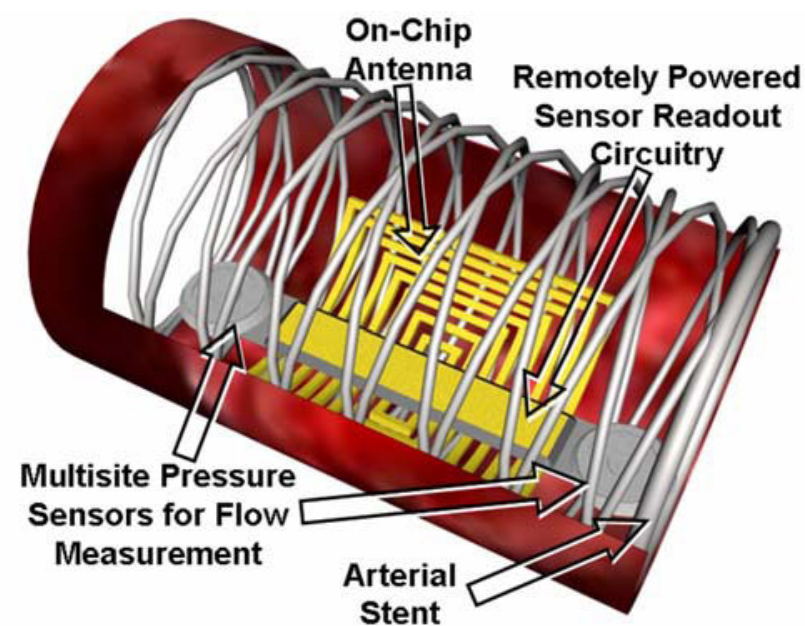

Figure 1. Illustration of the implantable, wireless system embedded inside an arterial stent. blood flow when diagnosing patients. Acute measurement of blood flow can be performed through guide-wire tip instrumentation to either directly or indirectly measure the flow across an occlusion. One of the indirect methods of measuring flow is utilizing a multi-site pressure measurement system $[2,3]$. The complex relationship between pressure and flow is a result of the non-newtonian fluidic properties of blood and the elastic arterial walls. However, models that are not based on finite element modeling of arterial flow still use the Navier-Stokes formulas to build an equivalent circuit model for the relationship. The Navier-Stokes formula defines the differential pressure to distance relationship as

$$
-\frac{\Delta P}{\Delta x}=\left(\frac{8 \eta}{\pi R_{i}^{4}}\right) Q+\frac{\rho}{\pi R_{i}^{2}} \frac{d Q}{d t}
$$

where $Q$ is the flow rate, $\eta$ is the viscosity, $\rho$ is the density, and $R_{i}$ is the lumen radius. The first term defines the situation for constant flow and the second term adds the effects from a timevarying flow rate, which is the case in cardiovascular applications. The implementation of differential pressure measurement in diagnosing the cardiovascular system is shown in Figure 2.

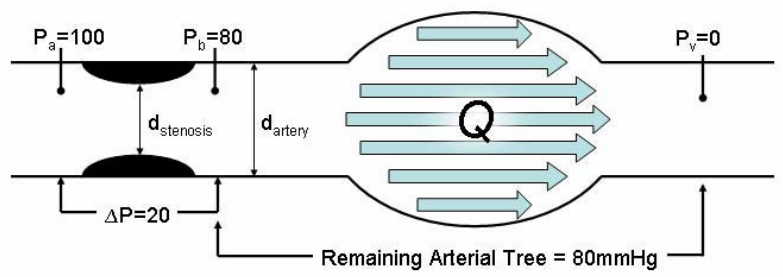

Figure 2. Illustration of the multisite pressure measurement enabling monitoring for arterial restenosis.

The narrowing of the artery from the build up of plaque between the two sensing sites causes a reduction of effective lumen radius from $d_{\text {artery }} / 2$ to $d_{\text {stenosis }} / 2$. As the narrowing progresses, a differential pressure measurement can be used to diagnose the percent stenosis of the artery defined by the ratio of $d_{\text {stenosis }} / d_{\text {artery. }}$. The differential pressure through the narrowed section can also indirectly measure a reduction in arterial flow.

\section{SYSTEM DESIGN}

The system interface is based on backscatter-modulated passive telemetry. This wireless scheme allows an implanted system to function from transcutaneous power delivered by an external system. The coupling of the external and internal antennae provides not only the power transfer but also the ability to sense the load on the wireless subsystem through the carrier waveform. Backscatter modulation is implemented by actively 
loading the secondary system which creates an amplitude modulation (AM) of the carrier signal [4]. An overview of the coupled telemetry system is shown in Figure 3. The external system is composed of both a power amplifier, which generates the carrier waveform, and envelope detector, which implements AM

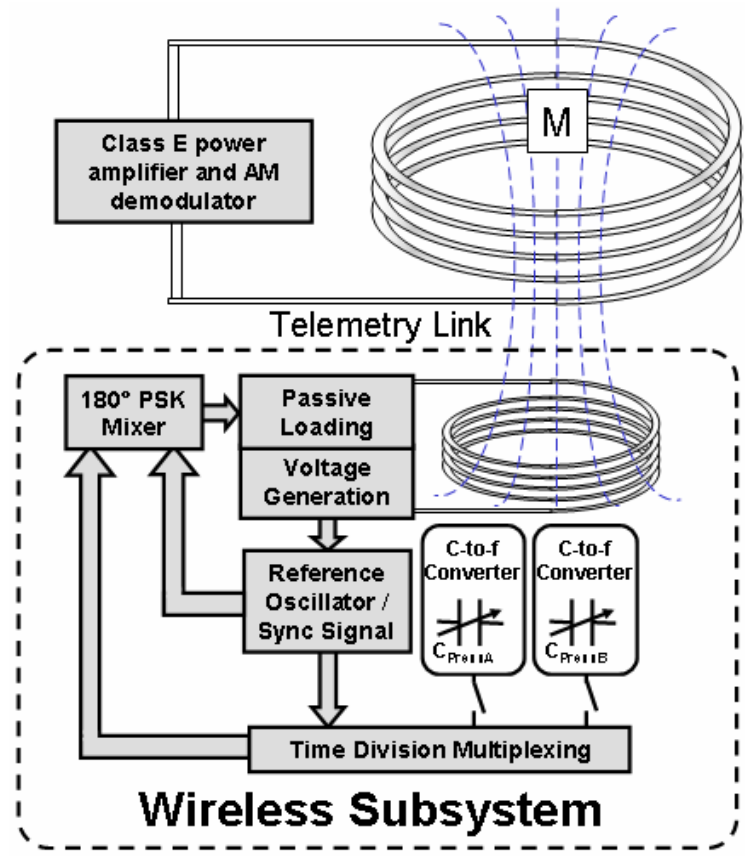

Figure 3. Schematic of the wireless systems showing the loosely coupled transformer system as well as the block diagram for the wireless subsystem.

demodulation of the carrier. The external system has been updated from previous systems that have implemented class $\mathrm{E}$ power amplifiers and AM envelope detectors that were developed for transcutaneous stimulation and RFID tags $[\mathbf{5}, \mathbf{6}]$. The power amplifier operates with a resonant tank at $4 \mathrm{MHz}$ with a quality factor of 100, which balances the tradeoffs between power efficiency and signal recovery bandwidth that is inherent in using the same antenna to simultaneously transmit and receive signals. The demodulator has a passband and carrier suppression of $30 \mathrm{~dB}$ between $1-30 \mathrm{kHz}$ and $-15 \mathrm{~dB}$ at $3 \mathrm{MHz}$, respectfully.

The wireless subsystem is composed of three sections: the RF front-end for power recovery and passive loading, the transducers and their interface circuitry, and the sensor and reference multiplexing and mixing. The RF front-end implements a fullbridge, bipolar rectifier along with a shunting transistor for resistive loading of the front-end LC resonant tank that has been previously presented [7]. This system, however, integrates the antenna along with the circuitry and uses 0201 surface mount capacitors for the front-end LC tank and power regulator. This implementation minimizes the area needed for active circuitry and minimizes the profile of the system

The capacitive pressure transducers are formed using a bulkmicromachined diaphragm along with the silicon-on-glass dissolved wafer process and are designed for a target pressure range between $700-900 \mathrm{mmHg}[\mathbf{8}]$. The technique used for vacuum sealing of the absolute transducers implements a silicon-gold eutectic and anodic bonding [9]. The Si-Au eutectic enables reflow of a dry etched feedthrough tunnel which is sealed during the anodic bonding process between the $\mathrm{Si}$ and glass substrates.

The sensor interface utilizes a capacitive-to-frequency converter implemented using a relaxation oscillator that is based on an oscillator implemented by Song, et al. [10]. Figure 4 shows the schematic of the relaxation oscillator along with the $V_{t}$ current source and SR flip-flop digitizer. With the frequency of the oscillator proportional to both the bias current and the sensor capacitance, the current sources can be designed to obtain the desired frequency range for the base capacitance of each sensor.

An on-chip reference oscillator is also implemented using

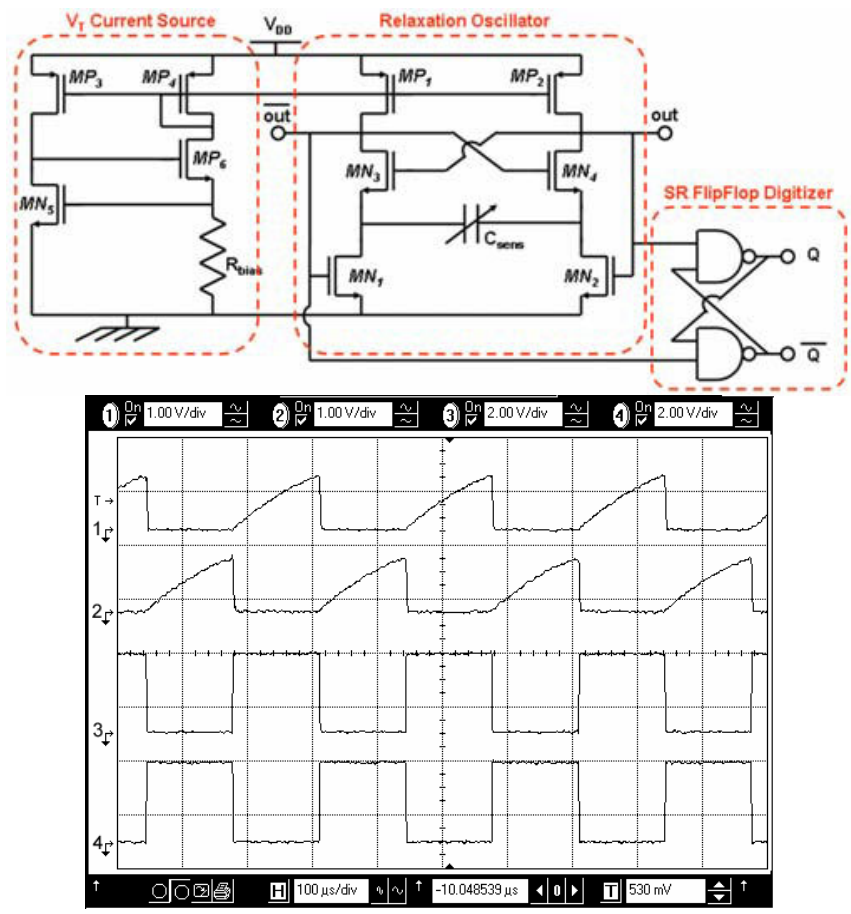

Figure 4. (upper) Schematic of the capacitance-tofrequency conversion circuitry. (lower) Oscilloscope measurement of the branches of the relaxation oscillator, (traces 1 and 2) and its digitized output (traces 3 and 4).

the same relaxation oscillator along with integrated poly-to-poly capacitors. This reference oscillator not only provides the ability to query the regulated power of the wireless device, but also the on-chip clock to control the multiplexing between the two transducers. Since backscatter modulation provides only a serial data stream between the two systems, a frequency mixer that combines the frequency content from the two sensors and the reference oscillator has been implemented. Figure 5 shows the block diagram of the mixer that time division multiplexes the twosensor interface oscillators and mixes in the reference oscillator frequency through phase-shift-keying (PSK) as well as the waveforms measured on the probe station before deposition of the antenna and anodic bonding of the sensors. The PSK modulated signal is then used to load the carrier signal. The amplitude modulation frequency then corresponds directly to the frequency information from the PSK signal, which contains the reference and

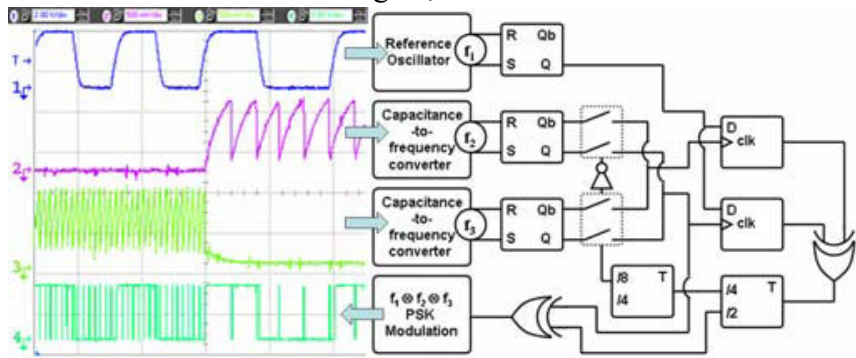

Figure 5. Schematic of the time-division multiplexed, phase shift keyed (PSK) frequency synthesizer along with oscilloscope waveforms. 
transducer information.

\section{INTEGRATED FABRICATION PROCESS}

The fabrication process for this system combines previous work that integrated boron diffused pressure sensors along with a $3 \mu \mathrm{m}$ BiCMOS process [11] with the combination anodic-eutectic bonding used to seal the cavity [9]. The fabrication steps are implemented in 22 masks with 8 masks for the transducer and 14 for the antenna and circuitry. A final cross section of this process is shown in Figure 6. Photographs of the monolithic device are

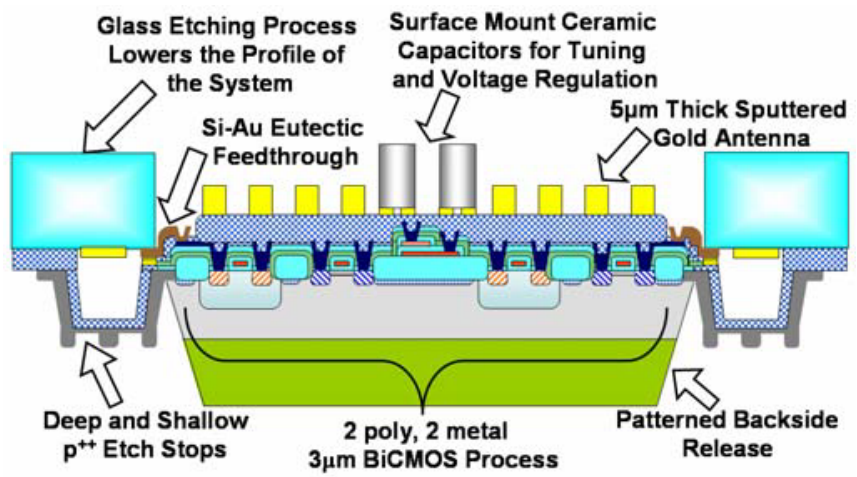

Figure 6. Cross section of the device showing the integration of the $3 \mu \mathrm{m}$ BiCMOS process with the silicon-onglass dissolved wafer process and an on-chip antenna.

shown in Figure 7. The on-chip antenna is realized in a $5 \mu \mathrm{m}$ thick gold layer that overlaps the circuit area and extends to form wings that will wrap around the surface of the artery or stent. Gold was used as the antenna material due to its high level of malleability to enable the curvature of the wings. The sensors are formed using consecutive deep and shallow boron diffusion after the p-well is implanted and the recess areas are etched. The n-epi/p-well CMOS process builds-in isolated, npn-bipolar transistors which are used in the RF front-end of the system. Since bulk silicon is bonded to glass through $3000 \AA$ of oxide, the glass is recessed to accommodate the topology from the CMOS and antenna features. After bonding, the backside of the silicon is patterned and etched using a Deep Reactive Ion Etcher (DRIE). This etch thins the antenna wings and sensor areas to approximately $80 \mu \mathrm{m}$ so that the EDP release etch will not reach the circuit area before the sensors

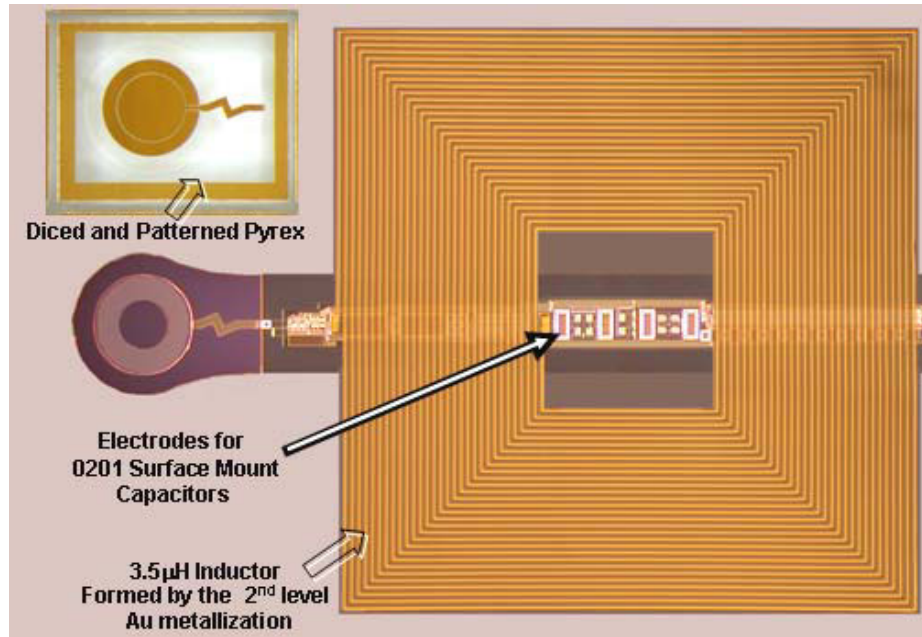

are defined and the wings are etched through to their underlying shallow boron diffusion.

After etching and metallization, the $500 \mu \mathrm{m}$ thick glass wafers are pre-diced to a depth of 4mils. Then, following the anodic bonding and DRIE etching of the silicon wafer, the backside of the glass is also diced along the same lines to a depth of 4 mils. The two wafer stack is mounted in wax on the silicon side and the backside of the glass wafer is etched in $49 \%$ hydrofluoric acid until the backside dice lines meet the front. This process not only thins the glass to approximately $100 \mu \mathrm{m}$, but also rounds the corners. This process is very sensitive to over etching since the HF will quickly undercut the wax and attack any exposed silicon after the glass punched through between the front side and back side dice lines. A cross-section and SEM shots of the glass thinning process are results are shown in Figure 8. The wafer is then removed from the wax mount and placed in EDP for release of the integrated devices. The only die level assembly needed for the system is the placement of the two surface-mount capacitors for power regulation and resonant tuning of the LC front-end. The devices are then encapsulated in parylene for electrical isolation during testing [1].
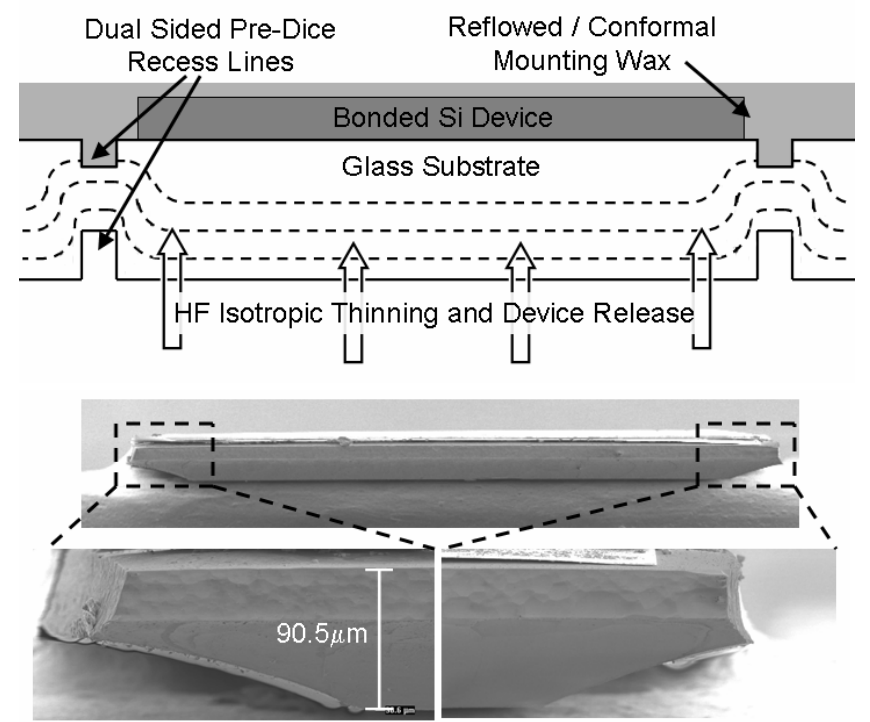

Figure 8. Cross section (upper) and SEM images (lower) showing the glass thinning process that is used to reduce the vertical footprint of the wireless system.

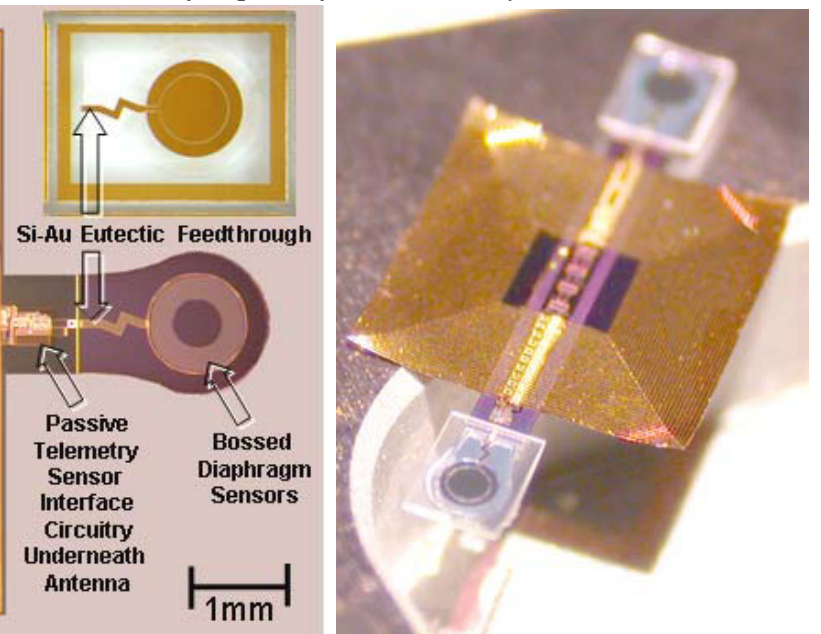

Figure 7. (left) Die photograph of the device before bonding showing the pressure sensors, interface circuitry, on-chip antenna and corresponding patterned glass electrode used for each capacitive transducer. (right) Photograph of the released, wireless system. 


\section{TEST RESULTS}

The passive telemetry interface circuit has a power consumption of $340 \mu \mathrm{W}$ from a $3 \mathrm{~V}$ supply. The capacitive pressure transducers have a sensitivity of $10 \mathrm{fF} / \mathrm{mmHg}$ after the parylene deposition and the c-to-f converter shows a response of $750 \mathrm{~Hz} / \mathrm{pF}$ at $10 \mathrm{kHz}$. Since analog frequency transmission is used for wireless readout, the resolution of the system is found through integration of the phase noise spectrum of the oscillator. This analysis finds a resolution of $25 \mathrm{~Hz}$ at a $100 \mathrm{~Hz}$ measurement bandwidth, which corresponds to a pressure resolution of $3 \mathrm{mmHg}$.

The test setup used for flow characterization utilized a rigid, $6 \mathrm{~mm}$ diameter piece of tubing and a voltage controlled gear pump to simulate flow within a stented carotid artery with deionized water. The wireless system is then remotely queried by the external system through the $1 \mathrm{~mm}$ wall of the tube. Pressure versus flow characterization of the test setup found a transducer sensitivity of $43(\mathrm{ml} / \mathrm{min}) / \mathrm{mmHg}$. Due to the large radius, the pressure drop across the $1 \mathrm{~cm}$ distance between the sensors is below the detection limit when no occlusion is present. The difference between the two sites based on the theory from Eqn (1)

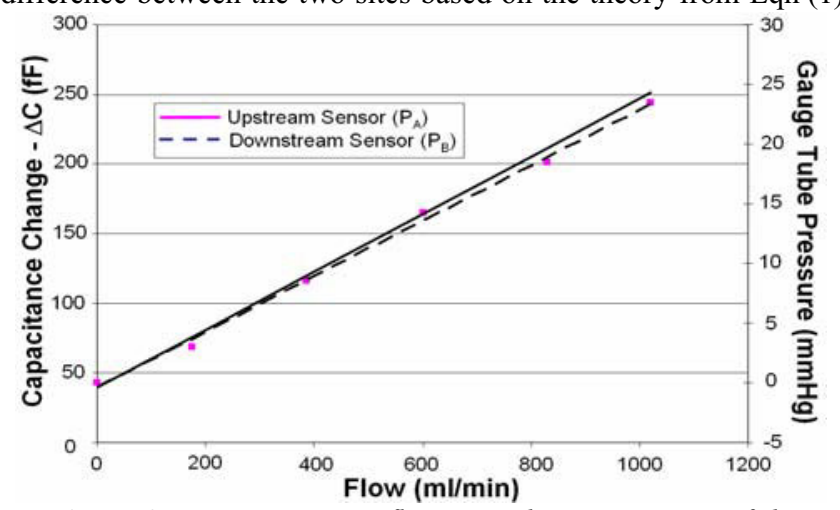

Figure 9. Pressure versus flow rate characterization of the test setup using the wireless system.

is a shown in Figure 9 along with the measured flow versus pressure results. In characterizing the system to sense the build up of plaque in an artery, the tube radius between the two sensing sites was reduced to restrict the flow and simulate the effects of arterial restenosis. The differential pressure measurements as the percent occlusion increases are shown in Figure 10 along with the theoretical results predicted from Eqn (1) as well as the corresponding reduction in flow. The measurements show that an occlusion of $60 \%$ can be sensed with this system which corresponds to a $13 \%$ reduction in arterial flow. This condition is similar to the qualifications for angioplasty intervention, which is

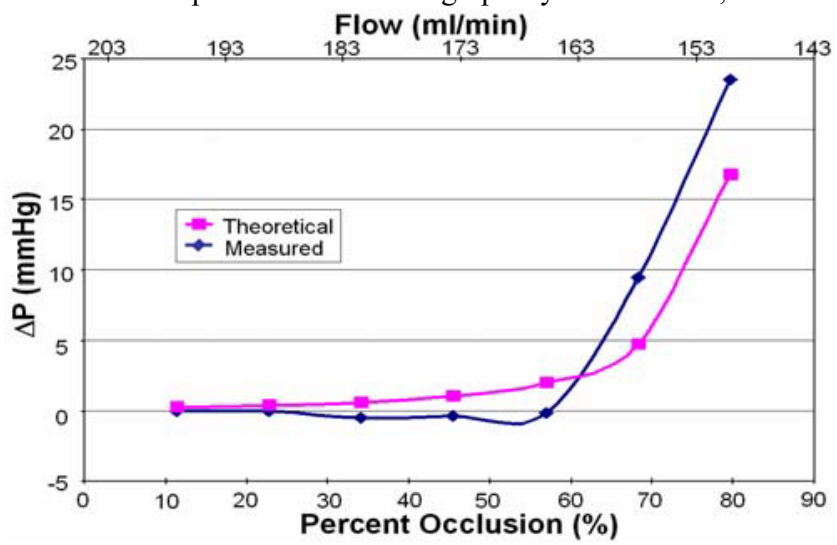

Figure 10. Pressure versus flow rate characterization of the system as the tube becomes occluded. an occlusion of $50 \%$ for the carotid artery [12].

\section{CONCLUSION}

The realization of a wireless arterial flow characterization system has been presented. The system uses two capacitive pressure transducers to monitor for stenosed restriction in arterial flow. The capacitance-to-frequency sensor interface oscillator has a sensitivity of $750 \mathrm{~Hz} / \mathrm{pF}$ and a base oscillation frequency of $10 \mathrm{kHz}$. A digital frequency synthesizer has been developed to provide asynchronous data transmission of the dual site pressure measurements, as well as, the reference signals through a backscattered modulation transmission scheme. The fabricated system has a volume of $2 \mathrm{~mm}^{3}$ and can detect a $13 \%$ reduction in peak arterial flow.

\section{ACKNOWLEDGMENTS}

This work is supported by the Engineering Research Centers Program of the National Science Foundation under Award Number EEC-9986866 and by a gift from Ms. Polly Anderson. The authors would like to thank Mayurachat Gulari for assistance in the BiCMOS fabrication process. Travel support has been generously provided by the Transducers Research Foundation and by the DARPA MEMS and DARPA BioFlips programs.

\section{REFERENCES}

[1] K. Takahata, et al., "A wireless microsensor for monitoring flow and pressure in a blood vessel utilizing a dual-inductor antenna stent and two pressure sensors," MEMS '04, Maastricht, Netherlands, 2004.

[2] J. Ji, et al., "An ultraminiature CMOS pressure sensor for a multiplexed cardiovascular catheter," IEEE Transactions on Electron Devices, vol. 39, pp. 2260-2267, 1992.

[3] N. H. J. Pijls and B. De Bruyne, Coronary Pressure. Boston: Kluwer Academic Publishers, 1997.

[4] K. Finkenzeller, RFID Handbook. Chichester, England: John Wiley \& Sons Ltd., 1999.

[5] T. Akin, "An integrated telemetric multichannel sieve electrode for nerve regeneration applications," in Electrical Engineering and Computer Science. Ann Arbor: University of Michigan, 1994.

[6] "MicroID 13.56MHz RFID System Design Guide," Microchip Technology Inc. 2001.

[7] A. DeHennis and K. D. Wise, "A passive-telemetry-based pressure sensing system," presented at Solid State Sensors and Actuators Workshop, Hilton Head, SC, 2002.

[8] A. V. Chavan and K. D. Wise, "Batch-processed vacuumsealed capacitive pressure sensors," IEEE Journal of MicroElectroMechanical Systems, pp. 580-588, 2001.

[9] A. DeHennis and K. D. Wise, "An all-capacitive sensing chip for temperature, absolute pressure, and relative humidity," Transducers '03, Boston, MA, 2003.

[10] B. Song, H. Kim, Y. Choi, and W. Kim, "A 50\% power reduction scheme for CMOS relaxation oscillator," presented at IEEE Asia-Pacific Conference on ASIC, Seoul, Korea, 1999.

[11] A. V. Chavan and K. D. Wise, "A monolithic fully-integrated vacuum-sealed CMOS pressure sensor," IEEE Transactions on Electron Devices, vol 49, pp. 164 - 169, Jan. 2002

[12] Y.-H. Tsai, et al.,"Angioplasty with stenting in treatment of carotid artery stenosis: report of a 3-year series," Chinese Journal of Radiology, vol. 28, pp. 361-366, 2003. 